Attitudes and Barriers to Healthcare

\section{M1 DEVELOPMENT OF A 'STOP-GO' SCREENING TOOL FOR STREAMLINING ASSESSMENT OF COMMON COMORBIDITIES IN COPD PATIENTS}

SL Kennie, HK Lamplough, EH Baker, S Mclvor. St George's University of London, London UK

\subsection{6/thoraxjnl-2016-209333.443}

Introduction and objectives People with COPD admitted to hospital with exacerbations have multiple co-morbidities, impacting on survival, re-hospitalisation and health status ${ }^{1}$. Although commonly described, there is no consensus coordinating management of COPD and co-morbidities in time- and resource-limited healthcare. We developed a pragmatic 'stop-go' pre-screening tool to streamline co-morbidity assessment and determined its efficacy in predicting co-morbid diagnoses made ad-hoc in COPD patients in the year following hospitalisation.

Methods Criteria were developed from NICE guidelines for twelve common COPD co-morbidities (Table). These indicated whether each condition was either not present or already diagnosed and treated (STOP - no further action required) or whether it could be present but undetected or could not be assessed (GO -further action required).

Electronic records (all patients) and hospital notes (84 patients) were reviewed for COPD patients admitted with exacerbations 1/9/2014-31/12/14 who survived for the subsequent year. The 'stop-go' screening tool was completed from admission data. Ad-hoc identification of new co-morbidities, defined by physician-diagnosis or commencement of new medication, was noted over 1 year follow up.

Results 120 patients (53\% male, 47\% female; age $73 \pm 10$ years) were included. The "stop-go "screening tool identified $7 \pm 2$ comorbidities per patient requiring no action and $5 \pm 2$ co-morbidities ('could be present', $2 \pm 1$; 'unable to assess' $3 \pm 3$ ) where further action was required. Information from patient records was generally insufficient to screen for anxiety and depression and lacking for around one quarter of patients for atrial fibrillation, diabetes mellitus and cognitive impairment.

During 1 year follow-up, patients developed $0.6 \pm 0.9$ (range 0-3) new diagnoses from the co-morbidities list. The 'stop-go' tool was most effective at predicting ad hoc diagnosis of hypertension, heart failure and osteoporosis (table) and less effective at predicting atrial fibrillation and ischaemic heart disease.

Conclusions The 'stop-go' pre-screening tool has potential to cut down the number of co-morbidities that need to be assessed in time-limited consultations. This could be improved by inclusion of anxiety and depression scoring, routine ECG and HbA1c. It shows early promise in predicting ad-hoc diagnoses, but now needs to be tested prospectively towards improving systematic and cohesive care provision.

\section{REFERENCE}

1 Public Health Reviews. 2011;32:451-474.

Abstract M1 Table 1 Identification of co-morbidities requiring further assessment using the 'stop-go' tool and relationship between screen results and actual co-morbidities identified by ad hoc healthcare contact over 1 year

\begin{tabular}{|c|c|c|c|c|c|c|c|}
\hline & \multicolumn{2}{|l|}{ STOP } & \multicolumn{2}{|l|}{ GO } & \multicolumn{3}{|c|}{ New diagnoses at 1 year } \\
\hline & $\begin{array}{l}\text { Screen negative } \\
\mathrm{n}(\%)\end{array}$ & $\begin{array}{l}\text { Prior diagnosis } \\
\text { n (\%) }\end{array}$ & $\begin{array}{l}\text { Screen positive } \\
\text { n (\%) }\end{array}$ & $\begin{array}{l}\text { Data not available } \\
\text { n (\%) }\end{array}$ & $n$ & $\begin{array}{l}\text { Go } \\
n(\%)\end{array}$ & $\begin{array}{l}\text { Stop } \\
\mathrm{n}(\%)\end{array}$ \\
\hline Hypertension & 20 & 62 & 23 & 15 & 12 & 11 & 1 \\
\hline$(B P<130 / 90)$ & (17) & $(52)$ & (19) & (13) & & $(92)$ & (8) \\
\hline Atrial Fibrillation & 71 & 21 & 1 & 27 & 7 & 3 & 4 \\
\hline (Sinus rhythm on ECG) & (59) & (18) & (1) & $(23)$ & & (43) & $(57)$ \\
\hline Ischaemic heart disease & 44 & 50 & 6 & 20 & 7 & 4 & 3 \\
\hline (No CVS symptoms) & (37) & $(42)$ & (5) & (17) & & $(57)$ & $(43)$ \\
\hline Congestive cardiac failure & 39 & 29 & 33 & 19 & 6 & 5 & 1 \\
\hline (BNP <100 pg/ml) & (33) & $(24)$ & $(28)$ & $(16)$ & & (83) & $(17)$ \\
\hline Dyslipidaemia & 11 & 64 & 27 & 18 & 2 & 1 & 1 \\
\hline (QRISK2 score <10\%) & (9) & (53) & (23) & (15) & & $(50)$ & $(50)$ \\
\hline Diabetes mellitus & 60 & 22 & 9 & 29 & 7 & 4 & 3 \\
\hline$\left(\mathrm{HbA}_{1} \mathrm{C} \leq 42 \mathrm{mmol} / \mathrm{mol}\right)$ & $(50)$ & (18) & (8) & $(24)$ & & $(57)$ & $(43)$ \\
\hline Depression & 1 & 20 & 0 & 99 & 4 & 4 & 0 \\
\hline (HADS-D <10) & (1) & (17) & $(0)$ & $(83)$ & & $(100)$ & $(0)$ \\
\hline Anxiety & 1 & 6 & 0 & 113 & 7 & 7 & 0 \\
\hline$($ HADS-A <10) & (1) & (5) & $(0)$ & (94) & & $(100)$ & $(0)$ \\
\hline Cognitive impairment & 73 & 7 & 6 & 34 & 6 & 5 & 1 \\
\hline (AMTS $\geq 7$ ) & $(61)$ & (6) & (5) & $(28)$ & & (83) & (17) \\
\hline Osteoporosis & 9 & 18 & 82 & 11 & 11 & 11 & 0 \\
\hline (No history of fragility \# and no oral corticosteroids for $\geq 3$ months) & (8) & $(15)$ & $(68)$ & (9) & & $(100)$ & $(0)$ \\
\hline Chronic kidney disease & 99 & 3 & 17 & 1 & $N / A$ & $\mathrm{~N} / \mathrm{A}$ & N/A \\
\hline (eGFR $\geq 60 \mathrm{ml} / \mathrm{min} / 1.73 \mathrm{~m}^{2}$ at discharge) & $(83)$ & (3) & (14) & $(1)$ & & & \\
\hline Anaemia & 88 & 3 & 28 & 1 & $\mathrm{~N} / \mathrm{A}$ & $\mathrm{N} / \mathrm{A}$ & $\mathrm{N} / \mathrm{A}$ \\
\hline ( $\mathrm{Hb} \geq 120 \mathrm{~g} / \mathrm{L}$ at discharge) & (73) & (3) & $(23)$ & (1) & & & \\
\hline
\end{tabular}

AMTS (Abbreviated Mental Test Score), BNP (BB-type Natriuretic Pepetide),CVS (Cardiovascular system), eGFR (Estimated Glomerular Filtration Rate), HADS-A/D (Hospital Anxiety and Depression Scale), Hb (Haemoglobin), HbA1 (Glycated Haemoglobin), \# (fracture). 